\title{
Coupling-fed Frequency Agile Monopole Fluid Antenna
}

\author{
Huan Li, Cristina Borda Fortuny, Kin-Fai Tong, and Kai-Kit Wong \\ Department of Electronic and Electrical Engineering \\ University College London (UCL) \\ London, United Kingdom \\ k.tong@ucl.ac.uk
}

\begin{abstract}
A coupling-fed reconfigurable monopole fluid antenna is investigated. The proposed geometry separates the ionized fluid from the feed line to prevent leakage. The preliminary results show that the operating frequency of the antenna can be reconfigurable by changing the length of the ionized fluid through an external pump. The peak efficiency is about $75 \%$ antenna can
\end{abstract}

\section{INTRODUCTION}

Fluid antennas have attracted researchers' attention because of its good conformability, beneficial re-configurability, small radar cross and convenience in transport. Nowadays, there are various kinds of liquid material can be used as the fluid antenna, such as fresh water [1], eutectic gallium indium (EGaIn) [2], [3], and ionized liquid[4], [5], [6]. Sodium Chloride ( $\mathrm{NaCl}$ ) dissolved water isconsidered as one of the most convenient fluid for antenna design that has the features of high permittivity, low cost, easier reconfiguration and eco-friendly characteristics compared to the liquid metal. And compared to the fresh water, the ionized fluid has better conductivity. Therefore, the ionized fluid becomes one of the popular materials in the fluid antenna design.

Typically, the ionized water is pumped through a certain container such as a tube, and the water will be pumped out continuously. Generally, the liquid in this type of antenna is controllable, where it is turned on if the liquid is flowing through the antenna, and it is turned off if the liquid is outflow from the substrate. But, these fluid antennas have the drawbacks of lower efficiency in communications and leakage when the fluid is pumped.

In this project, we propose a new design of the fluid antenna to overcome the drawbacks of the traditional fluid monopole antennas. The coupling feed antenna has the characteristics that it uses the feed itself to form the electromagnetic coupling in the antenna without any direct contact with the ionized fluid. Therefore, water leakage can be avoided.

3D-printer technology is used for fast and leakage proof container. Moreover, it gives the researchers more freedom in creating new shapes for fluid antennas.

Motivated by the above opportunity, this paper proposes to utilise low cost 3D-printed technology to design high efficiency monopole fluid antennas. CST microwave studio 2016 was used in the simulation [7].

\section{ANTENNA GEOMETRY}

The isometric and cross-sectioned views of the coupling-fed monopole fluid antenna is shown in Fig. 1. The antenna consists of three main parts, 1) a piece of $30 \times 30 \times 0.1 \mathrm{~mm}^{3}(\mathrm{~L} \times \mathrm{W} \times h) \mathrm{FR}$ 4 substrate with a ground plane on the bottom side, 2) a 3Dprinted circular PDMS tube containing the ionized fluid, and 3) a feed network which consists of a $50 \Omega$ microstrip feed line, vertical strip and a ring wrapped around circular tube.

The container tube for the ionised water is built using standard Clear Resin with Form 1+ Formlabs 3D printer. An experiment was set up to measure the parameters of the Clear Resin provided by Formlabs. The dielectric probe that was used is Agilent 85070E Dielectric Probe Kit. The dielectric constant measured is about 2.3, while the loss tangent is around 0.05 . Moreover, the molar concentration of the $\mathrm{NaCl}$ solution is 0.1 $\mathrm{mol} / \mathrm{L}$. The detailed dimensions of the antenna prototype can be found in Table 1
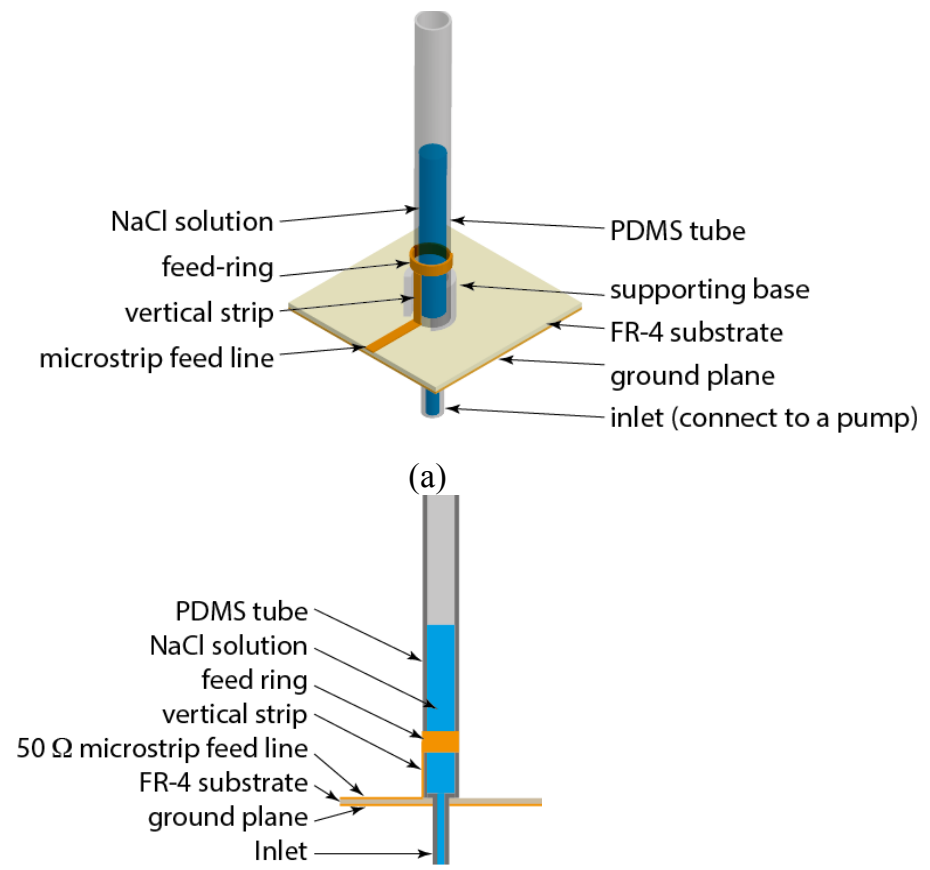

(b)

Fig. 1 (a) Isometric, and (b) cross-sectioned views of the coupling-fed monopole fluid antenna. 
TABle 1 Dimensions of The Coupling-Fed MonOpole FLUID ANTENNA.

\begin{tabular}{|c|c|c|}
\hline Parameters & Symbols & Dimensions (mm) \\
\hline Thickness of the tube & $h_{\text {tube }}$ & 0.7 \\
\hline Inner radius of the tube & $r_{\text {tube }}$ & 4.0 \\
\hline Radius of the inlet tube & $r_{\text {inlet }}$ & 1.0 \\
\hline $\begin{array}{c}\text { Length of the vertical strip from } \\
\text { the top of the FR-4 substrate }\end{array}$ & $y_{\text {strip }}$ & 7.0 \\
\hline Width of the vertical strip & $w_{\text {strip }}$ & 3.0 \\
\hline Thickness of the ring & $y_{\text {ring }}$ & 3.5 \\
\hline $\begin{array}{c}\text { Initial height of fluid from the } \\
\text { top of the tube base }\end{array}$ & $y_{\text {fluid }}$ & 5.0 \\
\hline
\end{tabular}

\section{RESULTS AND DISCUSSION}

The simulated and measured $S_{11}$ of the coupling-fed monopole fluid antenna at different of the fluid height $\left(y_{\text {fluid }}\right)$ is shown in Fig. 2. It can be observed that depending on the fluid height, the operating frequency of the antenna can be reconfigured between 3.5 to $6 \mathrm{GHz}$. To understand the radiation mechanism, the $\mathrm{H}$-field distribution of the antenna at $4 \mathrm{GHz}$ and is plotted in Fig. 3(a). It can be observed that at $4 \mathrm{GHz}$, the energy is radiated from the $\mathrm{NaCl}$ solution. Moreover, the donutshaped radiation pattern shown in Fig. 3(b) agrees with $\mathrm{H}$-field showing a classic radiation pattern of a monopole antenna. across the band. The maximum gain of the monopole is about 2 $\mathrm{dB}$ which mainly contributed by the fundamental mode of the fluid antenna. The peak efficiency of the antenna is about $80 \%$ when the fluid height is $5 \mathrm{~mm}$ and it drops to $60 \%$ if the height is increased to $25 \mathrm{mmm}$. This also explains the lower gain obtained at the higher frequency range.

In the study, it is observed that the base of the PDMS tube and the length of the feed probe play critical role in determining the efficiency. As the impedance of the antenna can be optimized by adjusting the height of the PDMS tube base, while the thickness of the feed ring will control the level of energy can be coupled to the fluid. In a preliminary study about the length of feed probe shows that the efficiency can be improved by proper selected thickness of the feed ring. Nevertheless, these two parameters should be carefully optimized for high efficiency. A parametric study will be presented in the final paper. Moreover, the experimental results will be provided in the presentation.

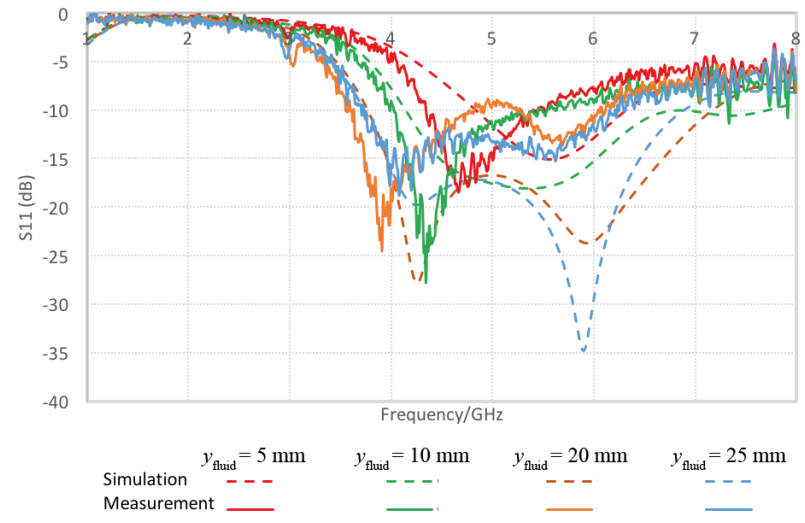

Fig. 2 Simulated and measured $\mathrm{S}_{11}$ of the proposed fluid antenna

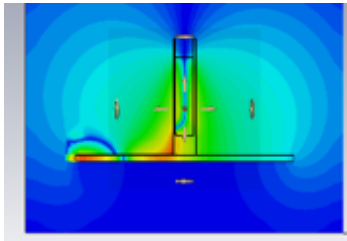

(a)

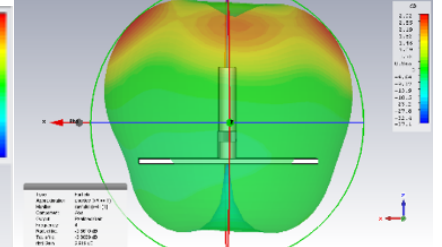

(b)
Fig. 3 (a) H-field, and (b) radation pattern of the proposed antenna at $4 \mathrm{GHz}$

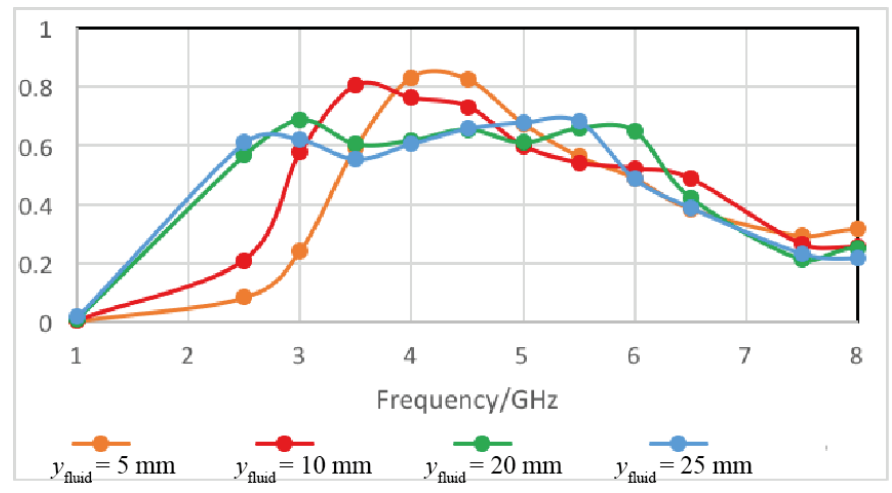

\section{CONCLUSION}

The preliminary study of a coupling-fed monopole fluid antenna has been presented. Reasonable frequency agility from 3.5 to $6 \mathrm{GHz}$ has been achieved and the peak efficiency is about $80 \%$. The radiation pattern of the antenna is stable across the operating bandwidth. Detailed study of the feed ring thickness and tube base height should be done to improve the efficiency at high frequency.

\section{REFERENCES}

[1] Y. Li and K. Luk, "A Water Dense Dielectric Patch Antenna," vol. 3, 2015.

[2] G. J. Hayes, J.-H. So, A. Qusba, M. D. Dickey, and G. Lazzi, "Flexible Liquid Metal Alloy (EGaIn) Microstrip Patch Antenna," TAP_IEEE Trans. Antennas Propag., vol. 60, no. 5, pp. 2151-2156, 2012.

[3] J. H. So, J. Thelen, A. Qusba, G. J. Hayes, G. Lazzi, and M. D. Dickey, "Reversibly deformable and mechanically tunable fluidic antennas," Adv. Funct. Mater., vol. 19, pp. 3632-3637, 2009.

Q. Xu, L. Xing, R. Alrawashdeh, Y. Shen, S. Al Ja' afreh, and Y. Huang, "Further investigation on water antennas," IET Microwaves, Antennas Propag., vol. 9, no. May 2014, pp. 735-741, 2015.

K. F. Tong, C. B. Fortuny, and J. Bai, "Low cost 3Dprinted monopole fluid antenna," in 2015 International Symposium on Antennas and Propagation (ISAP), 2015, pp. 1-4.

[6] C. Hua, Z. Shen, S. Member, and J. Lu, "HighEfficiency Sea-Water Monopole Antenna for Maritime Wireless Communications," vol. 62, no. 12, pp. 59685973, 2014.

[7] CST Microwave Studio - user manual. 
